\title{
Neuromyelitis optica and anti-aquaporin- 4 antibodies measured by an enzyme-linked immunosorbent assay
}

Sei Hayakawa, M.D. ${ }^{1)}$; Masahiro Mori, M.D. ${ }^{1)}$; Akiko Okuta, Ph.D. ${ }^{2)}$; Akiko Kamegawa, B.Sc. ${ }^{3,4)}$; Yoshinori Fujiyoshi, Ph.D. ${ }^{4,5)}$; Yasumasa Yoshiyama, M.D. ${ }^{6}$; Kaoru Mitsuoka, Ph.D. ${ }^{5)}$; Kenichi Ishibashi, M.D. ${ }^{7)}$; Sei Sasaki, M.D. ${ }^{8)}$; Takamichi Hattori, M.D. ${ }^{1)}$; Satoshi Kuwabara, M.D. ${ }^{1)}$

1) Department of Neurology, Graduate School of Medicine, Chiba University, Chiba, Japan

2) Japan Biological Information Research Center (JBIRC), Japan Biological Informatics Consortium (JBIC), Tokyo, Japan

${ }^{3)}$ Core Research for Evolution Science and Technology (CREST), Japan Science and Technology Agency (JST), Kyoto, Japan

4) Department of Biophysics, Faculty of Science, Kyoto University, Kyoto, Japan

5) Japan Biological Information Research Center (JBIRC), The National Institute of Advanced Industrial Science and Technology (AIST), Tokyo, Japan

${ }^{6)}$ Department of Neurology, Clinical Research Center, Chiba-East National Hospital, Chiba, Japan

${ }^{7)}$ Department of Medical Physiology. Meiji Pharmaceutical University, Tokyo, Japan

${ }^{8)}$ Department of Nephrology, Tokyo Medical and Dental University, Tokyo, Japan

Address correspondence to:

Masahiro Mori, MD

Department of Neurology, Graduate School of Medicine, Chiba University

1-8-1 Inohana, Chuo-ku, Chiba 260-8670, Japan

Tel. +81-43-222-2129 Fax. +81-43-226-2160

E-mail morim@faculty.chiba-u.jp

Word count: title, 11 (101 characters); abstract, 94; text, 3225. 


\begin{abstract}
NMO-IgG, a disease-specific autoantibody for neuromyelitis optica, recognizes aquaporin-4 (AQP4) and has been examined by indirect immunofluorescence assay. We developed an enzyme-linked immunosorbent assay (ELISA) to detect anti-AQP4 antibodies by establishing methods for expression in a baculovirus system and purification of recombinant AQP4 as antigen. Elevated anti-AQP4 antibody titers in serum were found in $15(71 \%)$ of 21 patients with neuromyelitis optica, $4.3 \%$ of 46 patients with multiple sclerosis, none of 51 normal controls, and $2.6 \%$ of 115 patients with other neurological diseases. The ELISA system can be substituted for the conventional NMO-IgG assay.
\end{abstract}

Keywords: Neuromyelitis optica; Multiple sclerosis; Aquaporin-4; Anti-aquaporin-4 antibody; ELISA

Abbreviations: AQP4, aquaporin-4; CSF, cerebrospinal fluid; EDSS, Expanded Disability Status Scale; ELISA, enzyme-linked immunosorbent assay; LESCL, long, extended spinal cord lesion; MS, multiple sclerosis; NMO, neuromyelitis optica; $\mathrm{NMO}^{\mathrm{AQP} 4}$, NMO defined by seropositivity for anti-AQP4 antibody; OD, optical density; OND, other neurological diseases; OSMS, optic-spinal multiple sclerosis; rAQP4, rat AQP4. 


\section{Introduction}

Neuromyelitis optica (NMO) (Devic's syndrome) is an inflammatory disease of the central nervous system clinically characterized by severe involvement of the optic nerves and spinal cord, and has been considered to be a disorder distinct from multiple sclerosis (MS) on the basis of clinical, imaging, pathological, and pathophysiological findings (Devic, 1894; Mandler et al., 1993; Wingerchuk et al., 1999; Wingerchuk and Weinshenker, 2003; Misu et al., 2006; Cabrera-Gómez et al., 2007; Hinson et al., 2007; Roemer et al., 2007).

Recently, NMO-IgG has been reported to be a novel serological marker for NMO (Lennon et al., 2004). NMO-IgG is an autoantibody that binds to constructs such as the microvessels, pia, subpia, and Virchow-Robin space at or near the blood-brain barrier. NMO-IgG labeling was performed using indirect immunofluorescence with a composite substrate of mouse tissues. In the original study, serum samples from 45 North American patients with NMO and 12 Japanese patients with the optic-spinal type of multiple sclerosis (OSMS) were studied (Lennon et al., 2004). The sensitivity and specificity of their assay were reported to be $73 \%$ and $91 \%$ for NMO, and $58 \%$ and $100 \%$ for OSMS in Japanese people, respectively.

Later, NMO-IgG was found to bind to the water channel protein aquaporin-4 (AQP4) (Lennon et al., 2005), a component of the dystroglycan protein complex that is densely expressed in astrocytic foot processes at the blood-brain barrier. We have developed an enzyme-linked immunosorbent assay (ELISA) with recombinant rat AQP4 as the antigen to directly detect serum anti-AQP4 antibody. The results were compared with those of the NMO-IgG assay.

\section{Materials and methods}

\subsection{Patients}


Consecutive Japanese patients who presented with NMO ( $n=21$; all women; mean age, 48.9 years) and those with MS ( $n=46 ; 33$ women and 13 men; mean age, 35.4 years) in our hospital between March 2000 and April 2006 were studied. NMO was diagnosed on the basis of the criteria of Wingerchuk et al. (2006). To compare anti-AQP4 antibody with NMO-IgG, we defined two types of NMO. NMO ${ }^{\mathrm{AQP} 4}$ was defined as cases fulfilling all items of the 2006 Wingerchuk criteria using seropositivity of anti-AQP4 antibody instead of NMO-IgG. Another type of NMO was defined by the 2006 Wingerchuk criteria excluding the NMO-IgG criterion. Patients with MS fulfilled McDonald's criteria for MS (McDonald et al., 2001; Polman et al., 2005).

Fifty-one healthy participants (34 women and 17 men; mean age, 37.1 years) served as the normal controls. A total of 115 patients with other neurological diseases (OND; 52 with Guillain-Barré or Fisher syndrome, 52 with myasthenia gravis, and 11 with miscellaneous neurological diseases, including encephalitis, myelitis, and myositis; 68 women and 47 men; mean age 49.1 years) served as disease controls. The medical records of the NMO and MS patients, including information about brain and/or spinal cord MRI scans recorded during clinical relapse, were reviewed by one of the authors (S.H.) blindly with respect to the outcome of serological assays. The clinical condition of the NMO or MS patients was monitored using the Expanded Disability Status Scale (EDSS) (Kurtzke, 1983). Using McDonald's criteria (McDonald et al., 2001; Polman et al., 2005), clinical relapse was defined as an episode of neurological disturbance, as seen in MS, lasting at least 24 hours. Patients who had selective involvement of the spinal cord and optic nerves but no clinical symptoms associated with cerebral, cerebellar, or brainstem involvement were classified as having OSMS. A lesion extending over more than three vertebral segments in a spinal cord MRI was defined as a long, extended spinal cord lesion (LESCL). Serum samples were obtained from all 67 patients with NMO or MS at the time of relapse and from 25 of these patients during 
remission. The remission phase was defined as the period when patients' neurological condition had been stable for more than 3 months and the next relapse did not occur for a further 3 months at least. Only 1 of the $46 \mathrm{MS}$ patients had received treatment with interferon $\beta$-1a from 5 years before the relapse; the other MS or NMO patients had received no treatment at the time of sampling of their sera in both the relapsing and remission phases. All the samples from the patients and healthy participants were stored at $-80^{\circ} \mathrm{C}$ until used and were numbered randomly by one of the authors (M.M.). Cerebrospinal fluid (CSF) samples were obtained from 20 patients with NMO and 44 with MS at the time of relapse.

NMO-IgG was measured for all the patients with NMO (21 patients) and MS (46 patients), but not for the normal or disease controls, at the Neuroimmunology Laboratory, Mayo Clinic, Rochester, MN, blindly with respect to clinical information and results of the ELISA assays for AQP4.

\subsection{Constructs, expression, and purification of rat AQP4}

The methods used for the expression and purification of rat AQP4 (rAQP4) were described previously (Hiroaki et al., 2006). Briefly, the baculovirus expression system was used to produce recombinant rAQP4. cDNA for shorter splicing variant rAQP4M23 (Fushimi et al., 1993) was used for the expression. Sf9 cells were grown at $27^{\circ} \mathrm{C}$ in $3-1$ spinner flasks, infected with $300 \mathrm{ml}$ of high-titer baculovirus with rAQP4 cDNA, and harvested 2 days later. Membranes were prepared from Sf9 cells as described previously (Doi et al., 1997). The membranes with rAQP4M23 were solubilized with 5\% (w/v) $n$-octyl- $\beta$,D-glucoside and purified with Ni-NTA agarose (Qiagen, Valencia, CA) (Hiroaki et al., 2006). The purified rAQP4M23 was stored at $-80^{\circ} \mathrm{C}$ until used.

\subsection{Anti-AQP4 antibody assay}


Serum anti-AQP4 antibody titer was measured by ELISA in duplicate using purified rAQP4 as the antigen by two of the authors (A.O. and A.K.), blindly with respect to clinical information and the results of NMO-IgG assays. The wells of ELISA plates (Nunc ImmobilizerTM Nickel Chelate F96; Nunc, Roskilde, Denmark) were coated with $50 \mu$ of 5 $\mu \mathrm{g} / \mathrm{ml} \mathrm{rAQP} 4$ in $0.7 \%$-octyl- $\beta$,D-glucoside, $20 \mathrm{mM}$ Tris- $\mathrm{HCl}(\mathrm{pH} 7.5)$, and $300 \mathrm{mM} \mathrm{NaCl}$, and incubated overnight at $4{ }^{\circ} \mathrm{C}$. The plates were washed with washing buffer $(0.2 \%$ Tween-20 in 10\% Block Ace [Dainippon Pharmaceutical, Osaka, Japan]) and blocked with blocking buffer $\left(25 \%\right.$ Block Ace) overnight at $4^{\circ} \mathrm{C}$. The plates were incubated for $60 \mathrm{~min}$ with $50 \mu \mathrm{l}$ of serum diluted 1:1000 in 10\% Block Ace, washed with washing buffer, and incubated for another $60 \mathrm{~min}$ at room temperature with peroxidase-conjugated goat anti-human IgG (Promega, Madison, WI) diluted 1:5000 in 10\% Block Ace. After another wash, the plates were developed with $50 \mu$ l of substrate solution (1-Step Ultra TMB-ELISA; Pierce, Rockford, IL) for $15 \mathrm{~min}$ at room temperature, and $50 \mu \mathrm{l}$ of $2 \mathrm{M} \mathrm{H}_{2} \mathrm{SO}_{4}$ was added to terminate the reaction. The optical density (OD) was measured at $450 \mathrm{~nm}$. The OD value obtained from the well without serum was subtracted from the value of each sample and was used for statistical analysis. Serum was considered to be anti-AQP4-positive if the OD was more than three standard deviations above the mean value for the 51 normal control samples.

\subsection{Direct competition ELISA}

To validate the specific binding of serum anti-AQP4 antibody to AQP4, a competitive inhibition assay was performed with a serum sample obtained from one of the anti-AQP4-positive NMO patients. The titer of serum anti-AQP4 antibody was measured using ELISA as described above. Fifty microliters of the serum, diluted 1:1000, was added to the wells together with $10 \mu \mathrm{l}$ samples of rAQP4 at various concentrations. The plate was incubated for 2 hours at room temperature. The titer was measured in duplicate. The OD value 
obtained from the well without serum was subtracted from the value of each sample and used for statistical analysis.

\subsection{Statistical analysis}

The significance of differences in percentages was determined by Fisher's exact probability test. Values for different groups were compared using the Mann-Whitney U test. The Wilcoxon signed-rank test was used to compare paired parameters. The relationship between anti-AQP4 antibody reactivities in NMO patients' sera and EDSS was estimated using the Spearman correlation coefficient test. Statistical analyses were performed using the SPSS statistical package (version 11.0.1J; SPSS Japan Inc., Tokyo, Japan). $P<0.05$ was considered significant. One of the authors (M.M.) conducted all the statistical analyses.

\section{Results}

\subsection{Anti-AQP4 antibody assay}

The results for the detection of serum anti-AQP4 IgG antibody by ELISA are shown in Fig. 1. Elevated serum anti-AQP4 antibodies were found in $15(71 \%)$ of the 21 patients with NMO, but not in any of the 51 healthy participants, and also in $2(4.3 \%)$ of the 46 patients with MS and 3 of the 115 (2.6\%) patients with OND (3 of 52 [5.8\%] patients with Guillain-Barré syndrome, none of 52 with myasthenia gravis, and none of 11 with miscellaneous neurological diseases) (Fig. 1A). The test for serum anti-AQP4 IgG antibody was thus more frequently positive in NMO patients than in the normal controls $(P<0.001)$ and OND patients $(P<0.001)$.

Figure 1B shows results of the anti-AQP4 ELISA in patient subgroups with and without the OSMS phenotype, NMO-IgG, and LESCL. Elevated serum anti-AQP4 antibodies were found in only $6(38 \%)$ of the 16 OSMS patients. In contrast, antibody positivity was found for 
$11(73 \%)$ of the 15 NMO-IgG-positive patients and $15(50 \%)$ of the 30 patients with LESCL. Thus, positivity for serum anti-AQP4 antibody was more frequent in patients with NMO-IgG than in those without it $(P<0.001)$, and in patients with LESCL than in those without it $(P<$ 0.001), whereas OSMS was not significantly associated with positivity for anti-AQP4 antibodies.

The levels of anti-AQP4 antibody in the serum of anti-AQP4 antibody-positive patients in the clinical relapse and remission phases are shown in Fig. 2A. After relapse, all nine patients received treatment with corticosteroids; six of them were treated with intravenous methylprednisolone infusion (1000 $\mathrm{mg}$ for 3 or 5 consecutive days), and the remaining three were given oral prednisolone for up to 21 days. The OD values during the period of relapse tended to be higher than those during remission $(P=0.055)$. The EDSS values of the anti-AQP4 positive patients were compared with the reactivities of anti-AQP4 antibody in their sera (Fig. 2B). The results suggested that the anti-AQP4 antibody reactivities in the anti-AQP4 positive patients' sera tended to be associated with their EDSS at the time of relapse when the serum samples had been obtained $(P=0.072)$.

\subsection{Direct competition ELISA}

Results of the direct competition ELISA with rAQP4 as competitor are shown in Fig. 3. Incubation with rAQP4 resulted in a dose-dependent decrease in the reactivity of anti-AQP4 antibody in the patient's serum.

\subsection{Clinical and laboratory characteristics of $N M O$ and $N M O^{A Q P 4}$ patients}

As described in Materials and methods, we defined two types of NMO: NMO and NMO ${ }^{\mathrm{AQP} 4}$. The clinical features of the NMO $(n=21), \mathrm{NMO}^{\mathrm{AQP} 4}(n=20)$, and MS patients $(n=46)$ are shown in Table 1 . The NMO and $\mathrm{NMO}^{\mathrm{AQP} 4}$ patients closely resembled each other in clinical 
characteristics. Compared with MS patients, they were older at the onset of disease, had a higher female-to-male ratio and EDSS, and had more frequent optic nerve involvement, severe, fixed attack-related weakness in one or more limbs, urinary retention, and transverse myelitis.

The laboratory features of the NMO $(n=21), \mathrm{NMO}^{\mathrm{AQP} 4}(n=20)$, and MS patients $(n=$ 46) are compared in Table 2. Again, the NMO and $\mathrm{NMO}^{\mathrm{AQP} 4}$ patients closely resembled each other. Compared with MS patients, they had a higher CSF cell count, more frequent CSF pleocytosis, higher CSF protein levels, more frequent LESCL, and more frequent normal brain MRI scans at the onset of disease (not meeting the criteria of Paty et al. (1988)), positive brain MRI at about the time of the last attack (meeting the criteria of McDonald et al. (McDonald et al., 2001; Polman et al., 2005)), and more frequent presence of NMO-IgG and anti-AQP4 antibody. Other laboratory tests considered were frequency of $\operatorname{IgG}$ index $>0.73$, frequency of the presence of oligoclonal bands, frequency of anti-nuclear, and anti-SS-A or anti-SS-B antibodies. None of the clinical and laboratory features were significantly different between $\mathrm{NMO}$ and $\mathrm{NMO}^{\mathrm{AQP} 4}$ patients.

\subsection{Correlation of anti-AQP4 ELISA with conventional NMO-IgG assay}

The correlation among three groups categorized according to NMO defined by the 2006 Wingerchuk criteria, and seropositivity for NMO-IgG or anti-AQP4 antibody is shown in Fig.

4. Twenty-four patients who had any of NMO, NMO-IgG, or anti-AQP4 antibody were included. Focusing on the association of NMO-IgG and anti-AQP4 antibody, 11 (73\%) of 15 the NMO-IgG-positive patients had anti-AQP4 antibody; conversely, 11 (65\%) of the 17 anti-AQP4-positive patients had NMO-IgG. For the diagnosis of NMO, 13 (62\%) of the 21 NMO patients were NMO-IgG-positive, and $15(71 \%)$ of the 21 NMO patients were anti-AQP4-positive. Therefore the sensitivity of anti-AQP4 antibody (71\%) was similar to 
that of NMO-IgG for NMO (62\%).

Three patients without NMO were positive for ELISA or NMO-IgG. One patient was positive for only ELISA, one was positive for only NMO-IgG, and the remaining patient was positive for both. The three patients had some of the clinical and laboratory features of NMO, such as recurrent severe optic neuritis and myelitis, recurrent myelitis with LESCL, and negative brain MRI at the onset, although they did not have LESCL, negative brain MRI at the onset, and optic neuritis.

Fifteen patients fulfilled the criteria for NMO excluding NMO-IgG seropositive status. Five $(33 \%)$ of them had both elevated anti-AQP4 antibody and NMO-IgG, five (33\%) had elevated anti-AQP4 antibody alone, two (13\%) had NMO-IgG alone, and the remaining three (20\%) had neither elevated anti-AQP4 antibody nor NMO-IgG. Therefore, for the diagnosis of NMO defined by the 2006 Wingerchuk criteria excluding NMO-IgG seropositive status, the sensitivity and specificity of the anti-AQP4 ELISA were $67 \%$ and $87 \%$, respectively, and those of the conventional NMO-IgG test were $47 \%$ and $85 \%$. Assuming that seropositive cases using the ELISA without NMO-IgG had relatively low titer compared with those with NMO-IgG (Fig. 1B), the sensitivity could be affected by these low titer cases. Moreover, because NMO-IgG was not measured in our OND group, the specificity of our ELISA and Mayo NMO-IgG could not be compared.

\section{Discussion}

We developed a new ELISA system that detects anti-AQP4 antibody in the serum. To the best of our knowledge, this is the first ELISA study of the detection of serum anti-AQP4 antibody in NMO. Using the ELISA, elevated anti-AQP4 antibodies were observed in $71 \%$ of the 21 Japanese NMO patients, which is a significantly higher percentage than in the MS patients, normal controls, and disease controls (Fig. 1A). 
The recombinant rat AQP4 protein used in our ELISA was an almost full-length protein. Since the three-dimensional structure of rAQP4 was analyzed using this exact construct (Hiroaki et al., 2006), the protein is known to be structurally intact and is therefore well suited to serve as an antigen for ELISA. The results of the competitive ELISA studies indicate that the anti-AQP4 antibody in patient's serum specifically reacts against AQP4 (Fig. 3). Since an ELISA-positive serum did not detect rAOP4 by immunoblot analysis (data not shown), detection of serum antibodies against AQP4 appears to require at least partially folded protein.

The titers of anti-AQP4 antibody tended to be higher at relapse than during clinical remission, as shown in Fig. 2A, and the titers at the relapse phase tended to correlate with the EDSS score, although there was no statistically significant difference between titers of anti-AQP4 antibody and EDSS score (Fig. 2B). All nine patients shown in Fig. 2A received short-term steroid therapy at relapse, resulting in a clinical improvement. Although we cannot exclude the possibility that the therapy affected the results independently of their disease activities, it is possible that the anti-AQP4 antibody titers correlated with disease activity. The quantitative analysis is an advantage of the ELISA system. The results support the view that anti-AQP4 antibodies may play a role in the pathogenesis of NMO and also suggest a molecular mechanism for the treatment of patients with NMO by plasma exchange (Watanabe et al., 2007).

The clinical and laboratory features of $\mathrm{NMO}^{\mathrm{AQP} 4}$ patients closely resembled those of NMO patients in this study (Tables 1 and 2) and were largely consistent with features of NMO in a study performed in the USA (Wingerchuk et al., 1999), OSMS in Japanese patients (Kuroiwa et al., 1975; Kira et al., 1996; Kira, 2003), NMO-IgG-positive Japanese patients with MS (Nakashima et al., 2006), and NMO (Matsuoka et al., 2007; Takahashi et al., 2007) (Tables 1 and 2).

NMO-IgG has been reported to specifically and sensitively bind to AQP4 (Lennon et al., 
2005). NMO-IgG was found in 33 out of 45 (73\%) North American patients with NMO based on the 1999 NMO criteria (Lennon et al., 2004). In these studies, NMO-IgG assays were performed on rat brain tissue using indirect immunofluorescence assay. Although NMO-IgG was found to bind rather selectively to the water channel protein AQP4 (Lennon et al., 2005), it is still possible that NMO-IgG binds not only AQP4 but also other antigens. Lennon et al. (2004) explained the seronegativity of NMO-IgG in NMO and OSMS patients by postulating that the use of rodent tissue rather than primate tissue caused reduced sensitivity in their indirect immunofluorescence assay. Recently, another sensitive assay for anti-AQP4 antibody in NMO patients has been reported (Matsuoka et al., 2007; Takahashi et al., 2007). In these studies, serum anti-AQP4 antibody was detected by indirect immunofluorescence assay using cell lines that either stably (Takahashi et al., 2007) or transiently (Matsuoka et al., 2007) expressed human AQP4. Since anti-AQP4 antibodies bound to the surface of AQP4-transfected cells in the absence of any membrane permeation process (Hinson et al., 2007; Matsuoka et al., 2007; Takahashi et al., 2007), the extracellular domains are regions detected by the anti-AQP4 antibody. While the amino acid sequences of rat and human AQP4 are highly homologous, there are some differences in the amino acid sequence in the extracellular loops (Jung et al., 1994). The recognition site of anti-AQP4 antibody has not been identified yet, and further studies are needed to identify it. The antigen used in our ELISA is rat AQP4, not human AQP4. A few differences in amino acid sequences between rats and humans may have caused the false-negative findings in our ELISA.

AQP4 is highly concentrated in astrocytic foot processes at the blood-brain barrier and is not restricted to the optic nerves or spinal cord (Jung et al., 1994; Lennon et al., 2004; Misu et al., 2006; Pittock et al., 2006). Moreover, it has been recently reported that NMO patients' serum IgG has a selective pathologic effect on cell membranes expressing AQP4 and targets astrocytic processes around the nodes of Ranvier, and that NMO lesions in CNS tissue 
demonstrate a striking loss of AQP4 regardless of the stage of demyelinating activity, which is different from the situation in multiple sclerosis (Misu et al., 2006; Hinson et al., 2007; Roemer et al., 2007). These characteristics indicate that the anti-AQP4 antibody is associated with impairment of blood-brain barrier function, thereby causing extensive severe lesions.

In conclusion, we established an ELISA-based test for antibodies against AQP4 and found that patients positive for the antibody constitute a distinct subgroup of MS patients whose characteristics are very similar to those of NMO and NMO-IgG-positive patients. The designation "OSMS," however, did not discriminate between anti-AQP4-positive and anti-AQP4-negative patient groups. ELISA can provide quantitative data to investigate correlations of antibody titers with disease severity and activity. Moreover, ELISA-based tests have the potential to be automated, which would make it possible to screen a large number of samples at a relatively low cost. The ELISA for anti-AQP4 antibody described here has the potential to replace or complement the currently used immunofluorescence test to detect NMO in order to further clarify the clinical features of NMO.

\section{Acknowledgments}

This research was supported by Grants in Aid for SPR, JST and NEDO, and in part by a grant for research on intractable diseases from the Ministry of Health, Labor and Welfare, Japan. 


\section{References}

Cabrera-Gómez, J.A., Quevedo-Sotolongo, L., González-Quevedo, A., Lima, S., Real-González, Y., Cristófol-Corominas, M., Romero-García, K., Ugarte-Sánchez, C., Jordán-González, J., de la Nuez, J.E., Lahera, J.G., Tellez, R., Pedroso-Ibañez, I., Roca, R.R., Cabrera-Núñez, A.Y., 2007. Brain magnetic resonance imaging findings in relapsing neuromyelitis optica. Mult. Scler. 13, 186-192.

Devic, E., 1894. Myélite subaiguë compliquée de névrite optique. Bull Med, Paris, 8, 1033-1034.

Doi, T., Hiroaki, Y., Arimoto, I., Fujiyoshi, Y., Okamoto, T., Satoh, M., Furuichi, Y., 1997. Characterization of human endothelin B receptor and mutant receptors expressed in insect cells. Eur. J. Biochem. 248, 139-148.

Fushimi, K., Uchida, S., Hara, Y., Hirata, Y., Marumo, F., Sasaki, S., 1993. Cloning and expression of apical membrane water channel of rat kidney collecting tubule. Nature. 361, 549-552.

Hinson, S.R., Pittock, S.J., Lucchinetti, C.F., Roemer, S.F., Fryer, J.P., Kryzer, T.J., Lennon, V.A., 2007. Pathogenic potential of IgG binding to water channel extracellular domain in neuromyelitis optica. Neurology. 69, 2221-2231.

Hiroaki, Y., Tani, K., Kamegawa, A., Gyobu, N., Nishikawa, K., Suzuki, H., Walz, T., Sasaki, S., Mitsuoka, K., Kimura, K., Mizoguchi, A., Fujiyoshi, Y., 2006. Implications of the aquaporin-4 structure on array formation and cell adhesion. J. Mol. Biol. 355, 628-639.

Jung, J.S., Bhat, R.V., Preston, G.M., Guggino, W.B., Baraban, J.M., Agre, P., 1994. Molecular characterization of an aquaporin cDNA from brain: candidate osmoreceptor and regulator of water balance. Proc. Natl. Acad. Sci. USA. 91, 13052-13056.

Kira, J., 2003. Multiple sclerosis in the Japanese population. Lancet Neurol. 2, 117-127.

Kira, J., Kanai, T., Nishimura, Y., Yamasaki, K., Matsushita, S., Kawano, Y., Hasuo, K., 
Tobimatsu, S., Kobayashi, T., 1996. Western versus Asian types of multiple sclerosis: immunogenetically and clinically distinct disorders. Ann. Neurol. 40, 569-574.

Kuroiwa, Y., Igata, A., Itahara, K., Koshijima, S., Tsubaki, T., 1975. Nationwide survey of multiple sclerosis in Japan: Clinical analysis of 1,084 cases. Neurology. 25, 845-851.

Kurtzke, J.F., 1983. Rating neurologic impairment in multiple sclerosis: an expanded disability status scale (EDSS). Neurology. 33, 1444-1452.

Lennon, V.A., Kryzer, T.J., Pittock, S.J., Verkman, A.S., Hinson, S.R., 2005. IgG marker of optic-spinal multiple sclerosis binds to the aquaporin-4 water channel. J. Exp. Med. 202, 473-477.

Lennon, V.A, Wingerchuk, D.M., Kryzer, T.J., Pittock, S.J., Lucchinetti, C.F., Fujihara, K., Nakashima, I., Weinshenker, B.G., 2004. A serum autoantibody marker of neuromyelitis optica: distinction from multiple sclerosis. Lancet. 364, 2106-2112.

Mandler, R.N., Davis L.E., Jeffery, D.R., Kornfeld, M.,1993. Devic's neuromyelitis optica: a clinicopathological study of 8 patients. Ann. Neurol. 34, 162-168.

Matsuoka, T., Matsushita, T., Kawano, Y., Osoegawa, M., Ochi, H., Ishizu, T., Minohara, M., Kikuchi, H., Mihara, F., Ohyagi, Y., Kira, J., 2007. Heterogeneity of aquaporin-4 autoimmunity and spinal cord lesions in multiple sclerosis in Japanese. Brain. 130, $1206-1223$

McDonald, W.I., Compston, A., Edan, G., Goodkin, D., Hartung, H.P., Lublin, F.D., McFarland, H.F., Paty, D.W., Polman, C.H., Reingold, S.C., Sandberg-Wollheim, M., Sibley, W., Thompson, A., van den Noort, S., Weinshenker, B.Y., Wolinsky, J.S., 2001. Recommended diagnostic criteria for multiple sclerosis: guidelines from the international panel on the diagnosis of multiple sclerosis. Ann. Neurol. 50, 121-127.

Misu, T., Fujihara, K., Nakamura, M., Murakami, K., Endo, M., Konno, H., Itoyama, Y., 2006. Loss of aquaporin-4 in active perivascular lesions in neuromyelitis optica: a case report. 
Tohoku J. Exp. Med. 209, 269-275.

Nakashima, I., Fujihara, K., Miyazawa, I., Misu, T., Narikawa, K., Nakamura, M., Watanabe,

S., Takahashi, T., Nishiyama, S., Shiga, Y., Sato, S., Weinshenker, B.G., Itoyama, Y., 2006.

Clinical and MRI features of Japanese patients with multiple sclerosis positive for

NMO-IgG. J. Neurol. Neurosur. Psychiatry. 77, 1073-1075.

Paty, D.W., Oger, J.J., Kastrukoff, L.F., Hashimoto, S.A., Hooge, J.P., Eisen, A.A., Eisen, K.A., Purves, S.J., Low, M.D., Brandejs, V., Robertson, W.D., Li, W.K.D., 1988. MRI in the diagnosis of MS: a prospective study with comparison of clinical evaluation, evoked potentials, oligoclonal banding, and CT. Neurology. 38, 180-185.

Pittock, S.J., Weinshenker, B.G., Lucchinetti, C.F., Wingerchuk, D.M., Corboy, J.R., Lennon, V.A., 2006. Neuromyelitis optica brain lesions localized at sites of high aquaporin 4 expression. Arch. Neurol. 63, 964-968.

Polman, C.H., Reingold, S.C., Edan, G., Filippi, M., Hartung, H.P., Kappos, L., Lublin, F.D. Metz, L.M., McFarland, H.F., O'Connor, P.W., Sandberg-Wollheim, M., Thompson, A.J., Weinshenker, B.G., Wolinsky, J.S., 2005. Diagnostic criteria for multiple sclerosis: 2005 revisions to the "McDonald criteria". Ann. Neurol. 58, 840-846.

Roemer, S.F., Parisi, J.E., Lennon, V.A., Benarroch, E.E., Lassmann, H., Bruck, W., Mandler, R.N., Weinshenker, B.G., Pittock, S.J., Wingerchuk, D.M., Lucchinetti, C.F. 2007. Pattern-specific loss of aquaporin-4 immunoreactivity distinguishes neuromyelitis optica from multiple sclerosis. Brain. 130, 1194-1205.

Takahashi, T., Fujihara, K., Nakashima, I., Misu, T., Miyazawa, I., Nakamura, M., Watanabe, S., Shiga, Y., Kanaoka, C., Fujimori, J., Sato, S., Itoyama, Y., 2007. Anti-aquaporin-4 antibody is involved in the pathogenesis of NMO: a study on antibody titre. Brain. 130, 1235-1243.

Watanabe, S., Nakashima, I., Misu, T., Miyazawa, I., Shiga, Y., Fujihara, K., Itoyama, Y., 
2007. Therapeutic efficacy of plasma exchange in NMO-IgG-positive patients with neuromyelitis optica. Mult. Scler. 13, 128-132.

Wingerchuk, D.M., Lennon, V.A., Pittock, S.J., Lucchinetti, C.F., Weinshenker, B.G., 2006. Revised diagnostic criteria for neuromyelitis optica. Neurology. 66, 1485-1489.

Wingerchuk, D.M., Weinshenker B.G., 2003. Neuromyelitis optica: clinical predictors of a relapsing course and survival. Neurology. 60, 848-853.

Wingerchuk, D.M., Hogancamp, W.F., O’Brien, P.C., Weinshenker, B.G., 1999. The clinical course of neuromyelitis optica (Devic's syndrome). Neurology. 53, 1107-1114. 


\section{Figure legends}

Fig. 1. ELISA results for serum anti-AQP4 IgG antibodies. (A) Serum anti-AQP4 antibody titers in each patient group. (B) Distribution of serum anti-AQP4 antibodies in serum samples of patients with (+) or without (-) OSMS, NMO-IgG, and LESCL. Patients diagnosed as NMO and MS are indicated by closed $(\diamond)$ and open $(\diamond)$ diamonds, respectively. The dashed line indicates the cut-off value determined from the data for the normal controls (3 SD above the normal mean value). NC, normal controls; NMO, neuromyelitis optica; MS, multiple sclerosis; OND, other neurological diseases, including Guillain-Barré syndrome, myasthenia gravis, and miscellaneous diseases; OSMS, optic-spinal multiple sclerosis; LESCL, long, extended spinal cord lesion.

Fig. 2. Anti-AQP4 antibody titer and clinical profile. (A) Serum anti-AQP4 IgG antibody levels in anti-AQP4-positive patients during relapse and remission phases. There was a tendency toward a difference between values at relapse and remission $(P=0.055)$. Dashed line indicates the cut-off value. (B) Correlation between anti-AQP4 IgG antibody reactivities (absorbance at $450 \mathrm{~nm}$ ) in anti-AQP4-positive patients' sera and EDSS. Reactivities tended to correlate with EDSS $(P=0.072)$. EDSS, Expanded Disability Status Scale.

Fig. 3. Competition ELISA. Competitive inhibition of reactivity of serum anti-AQP4 antibody by incubation with various concentrations of rAQP4. Error bars indicate SEM.

Fig. 4. The relationship of NMO, NMO-IgG, and anti-AQP4 antibodies. Patients were categorized into the three groups according to seropositivity for NMO-IgG, assessment of anti-AQP4 antibody, and whether they met the criteria for NMO. NMO, neuromyelitis optica. 
(A)

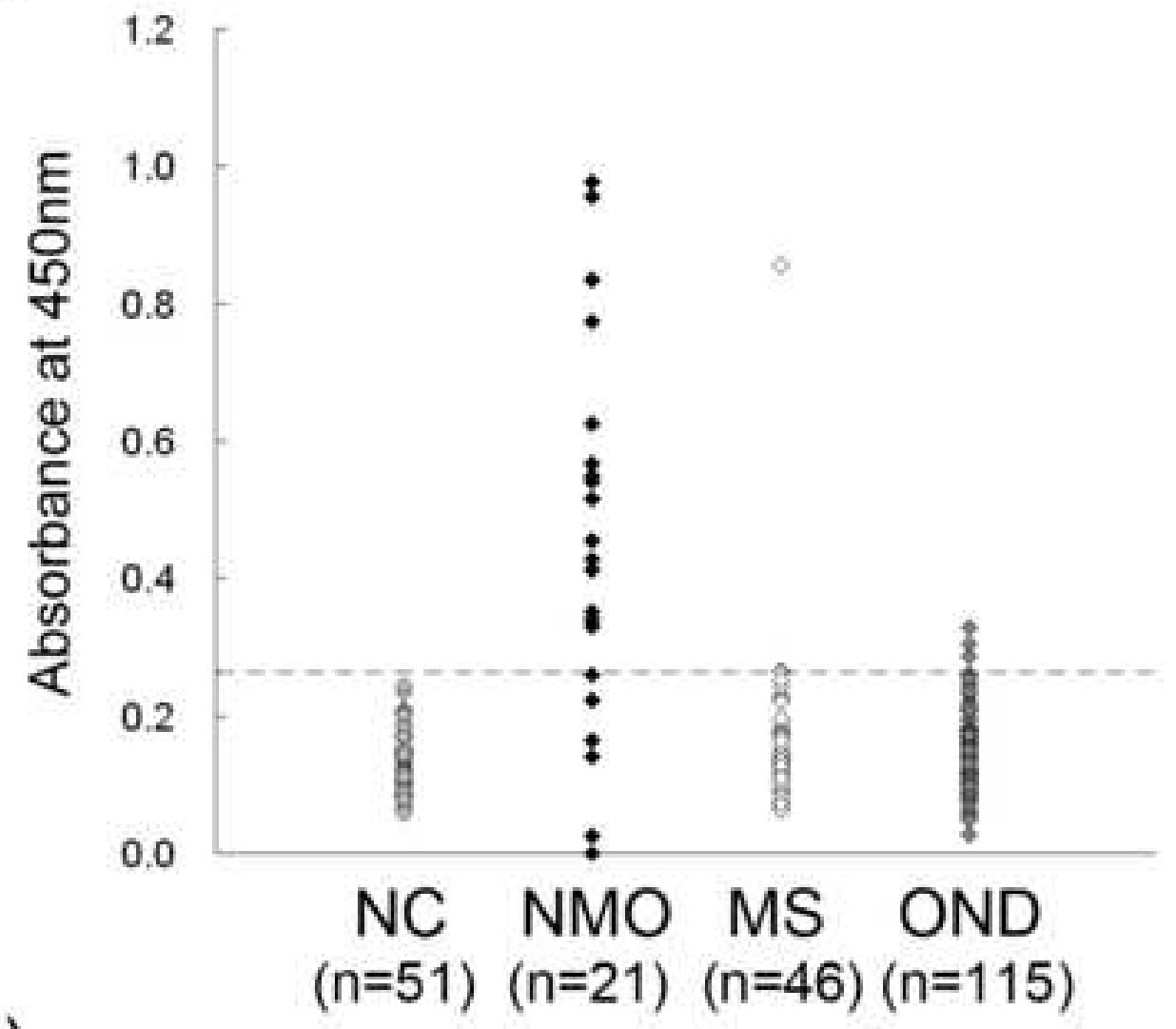

(B)

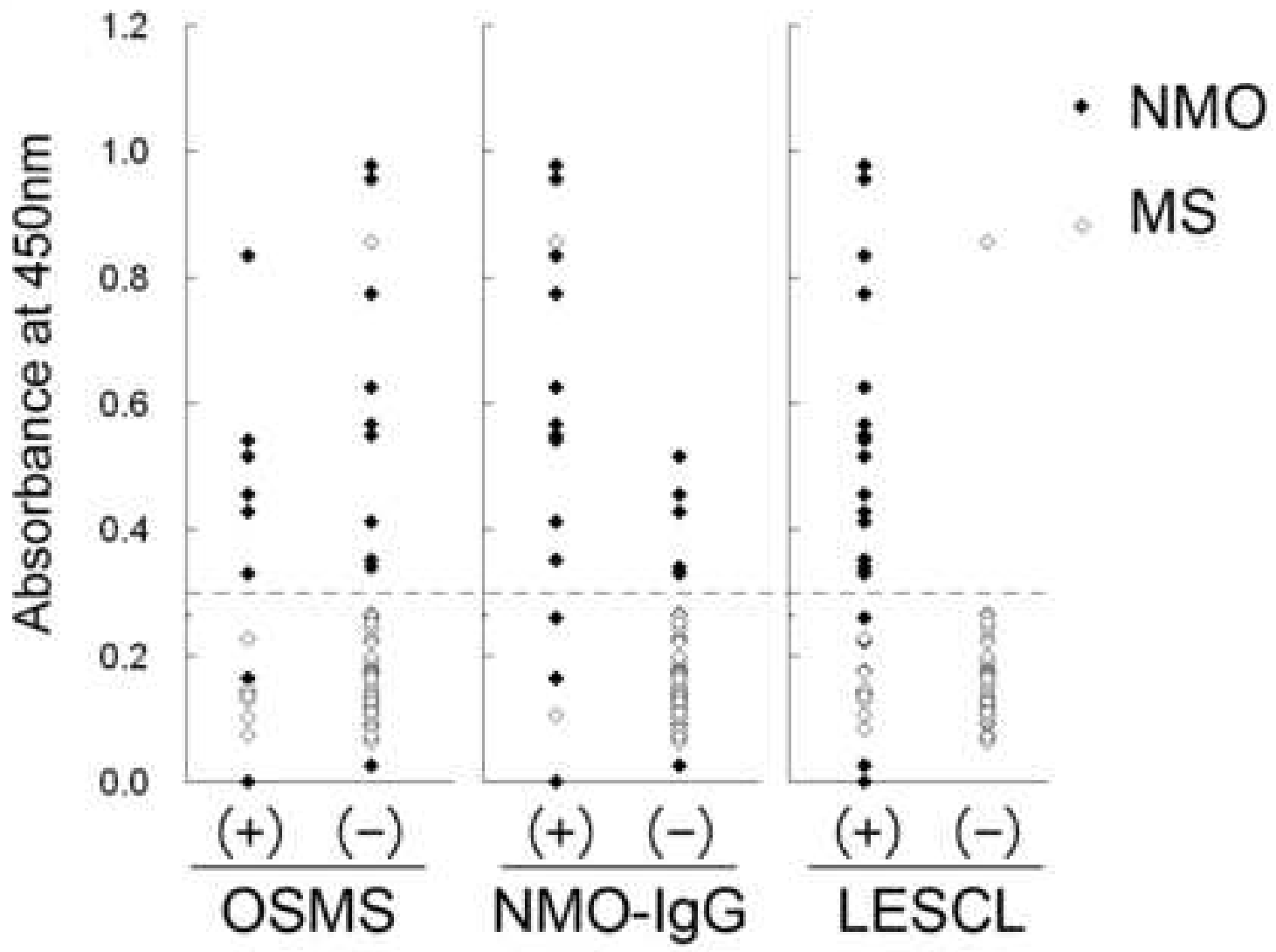




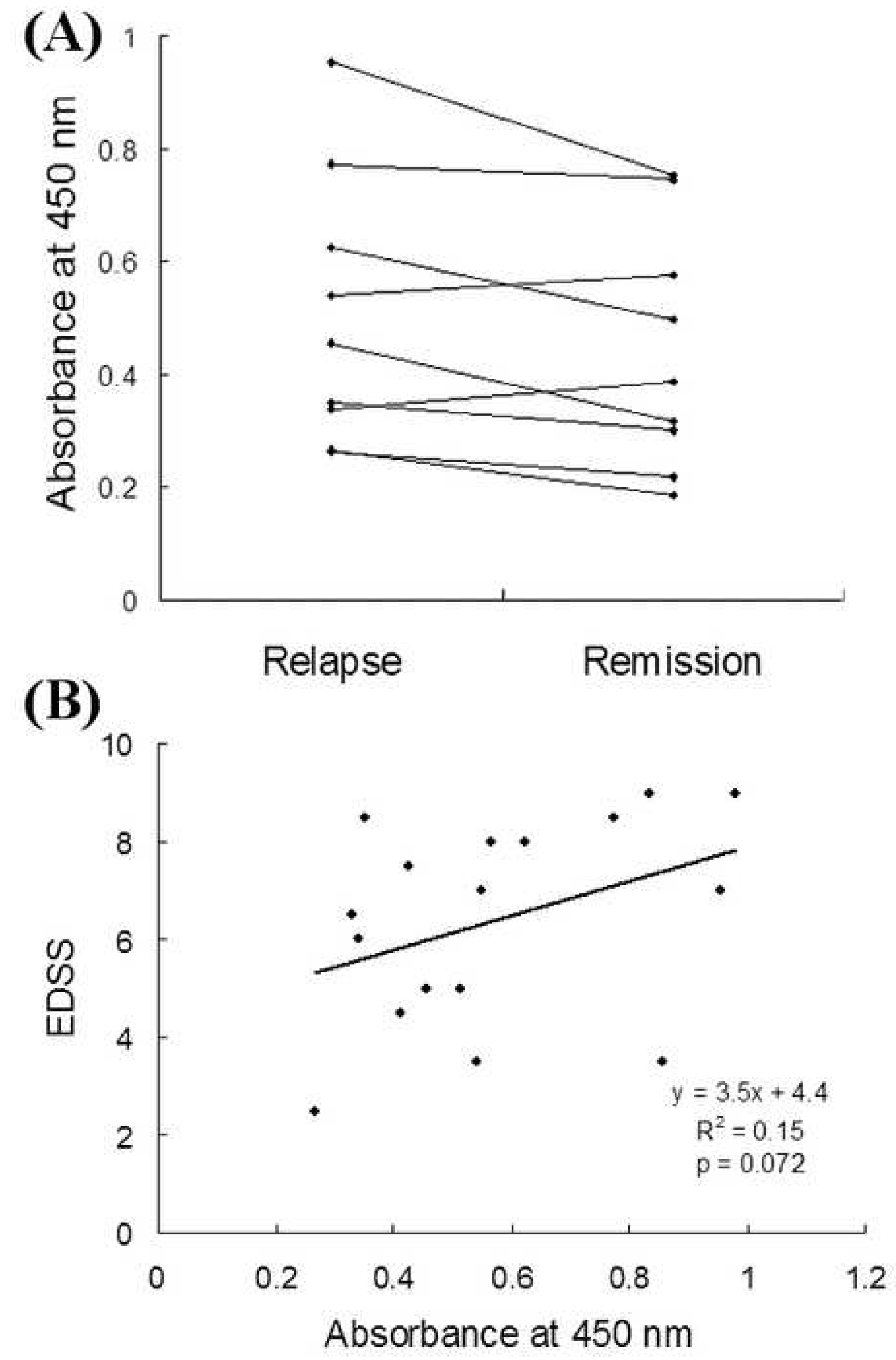

(B)

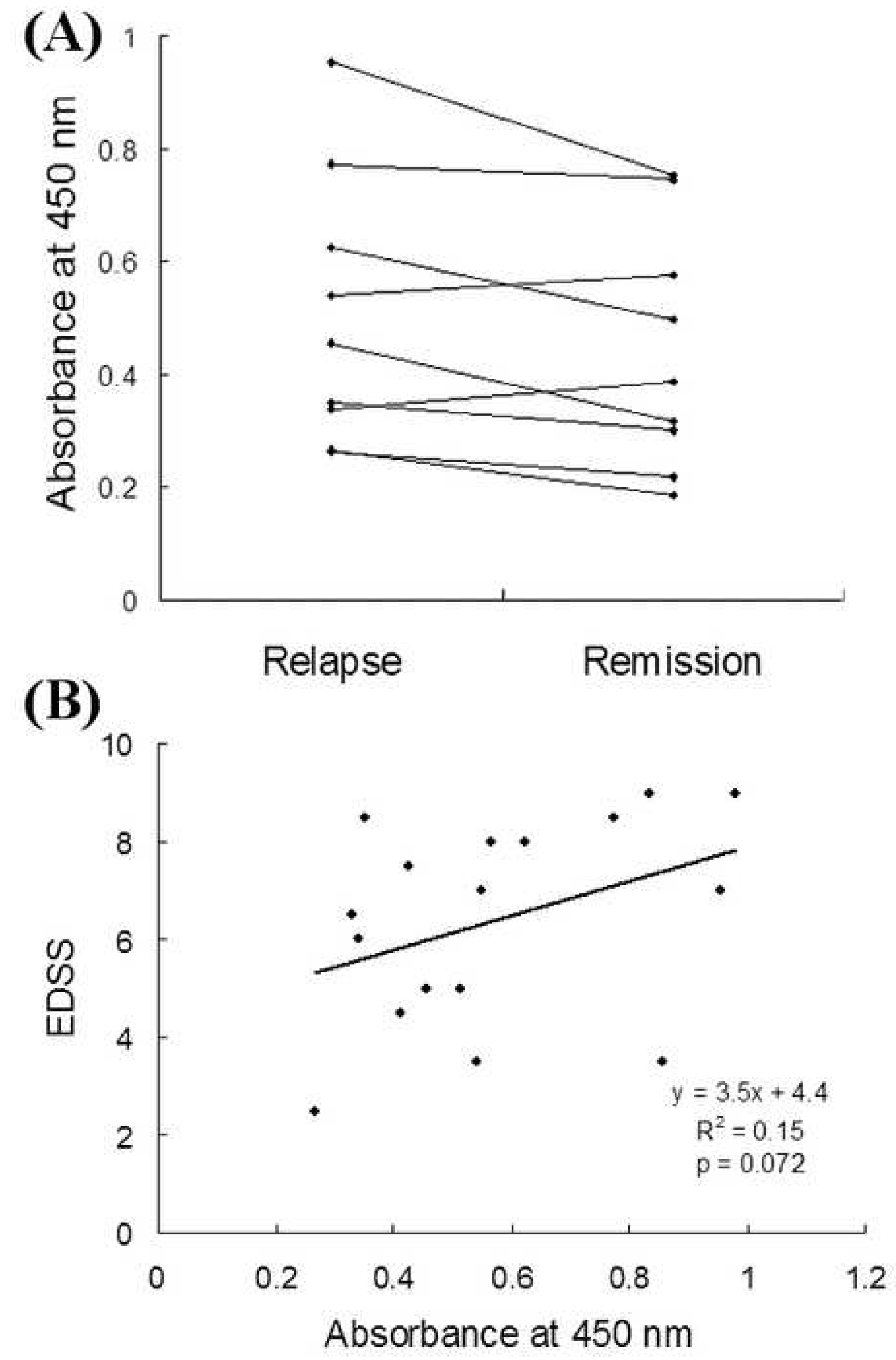

Figure 2 


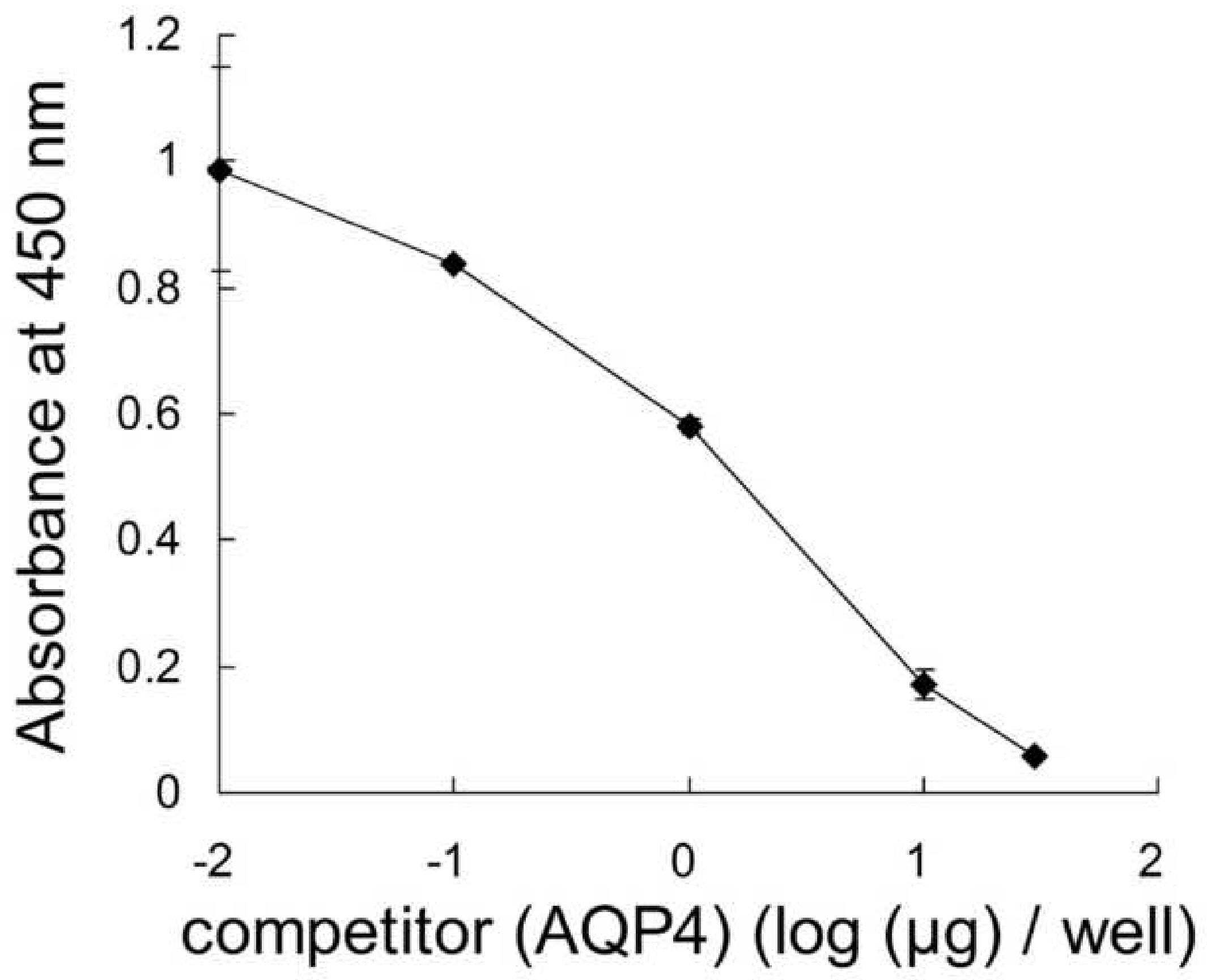




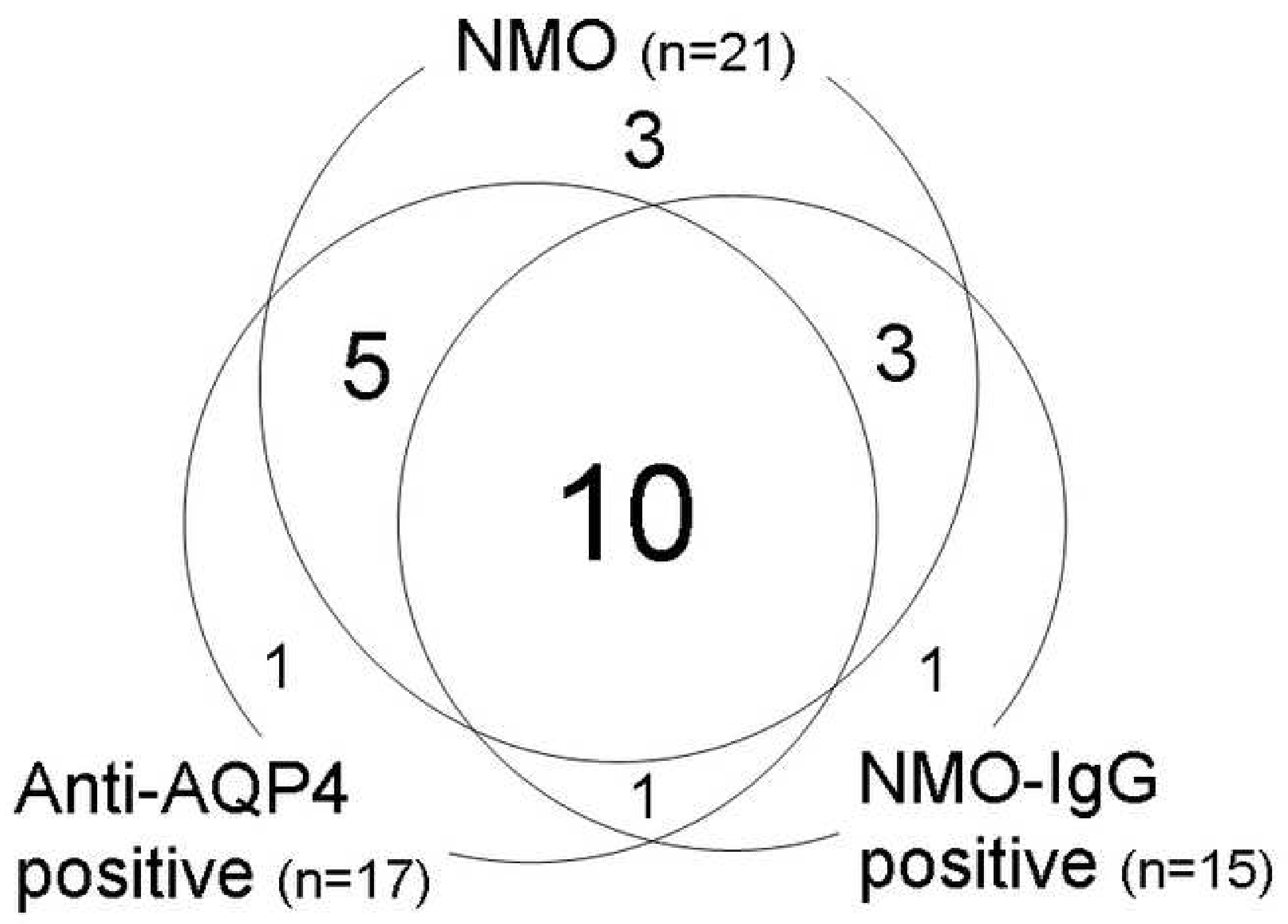


Table 1. Clinical features of NMO, $\mathrm{NMO}^{\mathrm{AQP} 4}$, and MS patients

\begin{tabular}{|c|c|c|c|c|c|c|}
\hline & \multirow{2}{*}{$\begin{array}{c}(\mathbf{A}) \\
\text { NMO } \\
(n=21)\end{array}$} & \multirow{2}{*}{$\begin{array}{c}\text { (B) } \\
\mathrm{NMO}^{\mathrm{AQP} 4} \\
(\mathrm{n}=20)\end{array}$} & \multirow{2}{*}{$\begin{array}{c}\text { (C) } \\
\text { MS } \\
(\mathrm{n}=46)\end{array}$} & \multicolumn{3}{|c|}{$P$-value } \\
\hline & & & & (A) vs (B) & $(\mathrm{A})$ vs $(\mathrm{C})$ & (B) vs (C) \\
\hline$\overline{\text { Age at onset }(\text { mean } \pm \text { SD) }}$ & $37.1 \pm 9.7$ & $48.9 \pm 11.6$ & $28.3 \pm 9.2$ & N.S.* & $<0.001^{*}$ & $<0.001^{*}$ \\
\hline Men : Women & $0: 21$ & $0: 20$ & $13: 33$ & N.S. & 0.006 & 0.006 \\
\hline Disease duration $(\mathrm{y}$, mean $\pm \mathrm{SD})$ & $11.7 \pm 10.2$ & $11.8 \pm 10.4$ & $7.0 \pm 6.3$ & N.S.* & N.S.* & N.S.* \\
\hline EDSS (median, range) & $7.0(3.0-9.0)$ & $7.3(3.0-9.0)$ & $3.5(0.0-9.0)$ & N.S.* & $<0.001^{*}$ & $<0.001 *$ \\
\hline \multicolumn{7}{|l|}{ Neurological system involved clinically; } \\
\hline Optic nerve & $21 / 21(100 \%)$ & $20 / 20(100 \%)$ & $25 / 46(54 \%)$ & N.S. & $<0.001$ & $<0.001$ \\
\hline Cerebrum & $7 / 21(33 \%)$ & $7 / 20(35 \%)$ & $15 / 46(33 \%)$ & N.S. & N.S. & N.S. \\
\hline Brainstem & $10 / 21(48 \%)$ & $10 / 20(50 \%)$ & $30 / 46(65 \%)$ & N.S. & N.S. & N.S. \\
\hline Cerebellum & $2 / 21(10 \%)$ & $2 / 20(10 \%)$ & $7 / 46(15 \%)$ & N.S. & N.S. & N.S. \\
\hline Spinal cord & $21 / 21(100 \%)$ & $20 / 20(100 \%)$ & $41 / 46(89 \%)$ & N.S. & N.S. & N.S. \\
\hline \multicolumn{7}{|l|}{ Clinical type; } \\
\hline Optic-spinal form & $8 / 21(38 \%)$ & $7 / 20(35 \%)$ & $8 / 46(17 \%)$ & N.S. & N.S. & N.S. \\
\hline \multicolumn{7}{|l|}{ Symptoms; } \\
\hline V.A. fixed at $20 / 200$ in at least one eye & $13 / 21(62 \%)$ & $13 / 20(65 \%)$ & $11 / 46(24 \%)$ & N.S. & 0.005 & 0.002 \\
\hline Bilateral optic neuritis simultaneously & $4 / 21(19 \%)$ & $4 / 20(20 \%)$ & $3 / 46(6.5 \%)$ & N.S. & N.S. & N.S. \\
\hline MRC grade $\leq 2$ for at least one limb & $13 / 21(62 \%)$ & $13 / 20(65 \%)$ & $8 / 46(17 \%)$ & N.S. & 0.001 & $<0.001$ \\
\hline Urinary retention & $14 / 21(67 \%)$ & $14 / 20(70 \%)$ & $14 / 46(30 \%)$ & N.S. & 0.008 & 0.006 \\
\hline Transverse myelitis & $16 / 21(76 \%)$ & $16 / 20(80 \%)$ & $9 / 46(20 \%)$ & N.S. & $<0.001$ & $<0.001$ \\
\hline
\end{tabular}

Fisher's exact probability test unless indicated; * Mann-Whitney U-test. NMO, neuromyelitis optica;

$\mathrm{NMO}^{\mathrm{AQP} 4}$, NMO defined by 2006 Wingerchuk criteria using the seropositivity of anti-AQP4 antibody, instead of NMO-IgG;

MS, multiple sclerosis; EDSS, expanded disability status scale; V.A., visual acuity; MRC, Medical Research Council 
Table 2. Laboratory features of $\mathrm{NMO}, \mathrm{NMO}^{\mathrm{AQP} 4}$, and MS patients

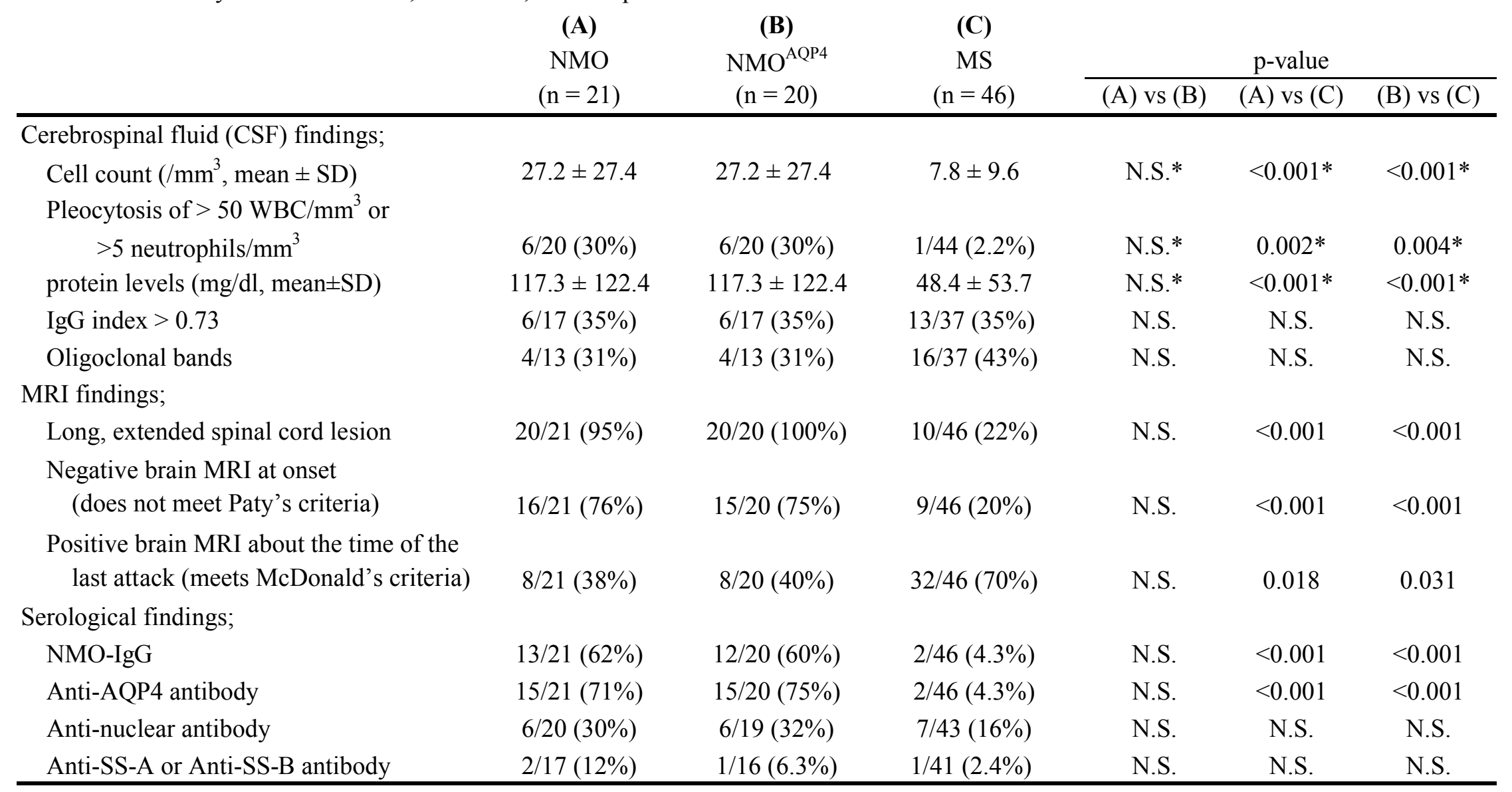

Fisher's exact probability test unless indicated; * Mann-Whitney U-test. NMO, neuromyelitis optica;

$\mathrm{NMO}^{\mathrm{AQP} 4}$, NMO defined by 2006 Wingerchuk criteria using the seropositivity of anti-AQP4 antibody, instead of NMO-IgG;

WBC, white blood cells; IgG, immunoglobulin G 